\title{
Meta Aircraft Flight Dynamics
}

\author{
Carlos Montalvo* and Mark Costelloı \\ Georgia Institute of Technology, Atlanta, Georgia 30332 \\ DOI: $10.2514 / 1 . C 032634$
}

\begin{abstract}
A meta aircraft is an air vehicle composed of a set of independent aircraft that are connected together in flight to form a larger composite aircraft. This paper explores the dynamics of meta aircraft systems with a focus on the changes in the aircraft flight dynamic modes and flexible modes of the system. Specifically, when aircraft are connected, basic flight dynamic modes such as the phugoid, short period, dutch roll, spiral, and roll modes change as a function of the number of connected aircraft. For aircraft connected in a wing-tip-to-wing-tip configuration, the longitudinal modes remain largely invariant with respect to the number of aircraft, whereas lateral modes are variable for both tip-to-tail and wing-tip-to-wing-tip connection configurations. In addition, connected aircraft exhibit complex flexible modes that change based on the characteristics of the connection joint and the number of connected aircraft. These conclusions are reached using a rigid six-degree-of-freedom representation for a single aircraft, where aircraft are connected through a set of linear and rotational elastic elements modeling the connection mechanism. Aerodynamic loads from all lifting surfaces are computed using a nonlinear lifting-line approach to enable aerodynamic wake interactions between connected aircraft.
\end{abstract}

\section{Nomenclature}

\begin{tabular}{|c|c|c|}
\hline$C_{L}, C_{D}$ & $=$ & lift and drag coefficients of aircraft \\
\hline$c$ & $=$ & mean chord of wing, $\mathrm{m}$ \\
\hline$I_{i}$ & $=$ & $\begin{array}{l}\text { mass moment inertia matrix about the } \\
\text { mass center in the body frame of the } \\
i \text { th aircraft, } \mathrm{kg} \cdot \mathrm{m}^{2}\end{array}$ \\
\hline$L_{C i}, M_{C i}, N_{C i}$ & $=$ & $\begin{array}{l}\text { total contact moment applied to } i \text { th } \\
\text { aircraft, } \mathrm{N} \cdot \mathrm{m}\end{array}$ \\
\hline$L_{i}, M_{i}, N_{i}$ & $=$ & $\begin{array}{l}\text { components of the total moment applied } \\
\text { to aircraft in body frame of the } \\
i \text { th aircraft, } \mathrm{N} \cdot \mathrm{m}\end{array}$ \\
\hline$L_{M i}, M_{M i}, N_{M i}$ & $=$ & $\begin{array}{l}\text { total magnetic moment applied to } \\
i \text { th aircraft, } \mathrm{N} \cdot \mathrm{m}\end{array}$ \\
\hline$m_{i}$ & $=$ & mass of $i$ th aircraft, $\mathrm{kg}$ \\
\hline$p_{i}, q_{i}, r_{i}$ & $=$ & $\begin{array}{l}\text { components of the mass center } \\
\text { angular velocity vector in the body } \\
\text { frame for the } i \text { th aircraft, rad/s }\end{array}$ \\
\hline $\boldsymbol{r}_{A \rightarrow B}$ & $=$ & $\begin{array}{l}\text { position vector from a generic point } \mathrm{A} \text { to } \\
\text { a generic point } \mathrm{B}, \mathrm{m}\end{array}$ \\
\hline$S$ & $=$ & reference area of wing, $\mathrm{m}^{2}$ \\
\hline$S(r)$ & $=$ & $\begin{array}{l}\text { skew symmetric matrix operator on a vector } \\
\text { (multiplying this matrix by a vector is } \\
\text { equivalent to a cross product) }\end{array}$ \\
\hline$u_{i}, v_{i}, w_{i}$ & $=$ & $\begin{array}{l}\text { components of the mass center velocity vector } \\
\text { in the body frame for the } i \text { th aircraft, } \mathrm{m} / \mathrm{s}\end{array}$ \\
\hline$V_{A B, C}$ & $=$ & $\begin{array}{l}\text { induced velocity at point } C \text { of a vortex } \\
\text { filament from point } A \text { to point } B, m / s\end{array}$ \\
\hline $\boldsymbol{V}_{A / B}$ & $=$ & $\begin{array}{l}\text { velocity vector of a generic point } A \text { with } \\
\text { respect to a generic frame } \mathrm{B}, \mathrm{m} / \mathrm{s}\end{array}$ \\
\hline$X_{C i}, Y_{C i}, Z_{C i}$ & $=$ & total contact force applied to $i$ th aircraft, $\mathrm{N}$ \\
\hline$X_{i}, Y_{i}, Z_{i}$ & $=$ & $\begin{array}{l}\text { components of the total force applied to } \\
\text { aircraft in body frame of the } i \text { th aircraft, } \mathrm{N}\end{array}$ \\
\hline$X_{M i}, Y_{M i}, Z_{M i}$ & $=$ & total magnetic force applied to $i$ th aircraft, $\mathrm{N}$ \\
\hline$x_{i}, y_{i}, z_{i}$ & $=$ & $\begin{array}{l}\text { components of the mass center position vector } \\
\text { in the inertial frame for the } i \text { th aircraft, } m\end{array}$ \\
\hline
\end{tabular}

Received 16 September 2013; revision received 3 March 2014; accepted for publication 4 March 2014; published online 11 June 2014. Copyright @ 2014 by the American Institute of Aeronautics and Astronautics, Inc. All rights reserved. Copies of this paper may be made for personal or internal use, on condition that the copier pay the $\$ 10.00$ per-copy fee to the Copyright Clearance Center, Inc., 222 Rosewood Drive, Danvers, MA 01923; include the code 1542-3868/14 and $\$ 10.00$ in correspondence with the CCC.

* Graduate Research Assistant, School of Aerospace Engineering. Member AIAA.

${ }^{\dagger}$ Professor, Guggenheim School of Aerospace Engineering, Woodruff School of Mechanical Engineering. Associate Fellow AIAA.

$$
\begin{array}{lll}
\Gamma_{j} & = & \text { strength of vortex filament at element } j \\
\rho & = & \text { atmospheric density, } \mathrm{kg} / \mathrm{m}^{3} \\
\phi_{i}, \theta_{i}, \psi_{i} & = & \text { Euler roll, pitch, and yaw of the } \\
& & i \text { th aircraft, rad }
\end{array}
$$

\section{Introduction}

M OBILE robotic systems are becoming a more common part of society. Future systems are envisioned to perform a wide variety of tasks, from ground vehicles that can automatically traverse a route to autonomous aircraft that can robotically takeoff, fly a mission, and land. For challenging missions, highly specialized robotic vehicles are needed for successful operation. Creation of specialized robots for a narrow task or mission can be both expensive and time-consuming. A solution to this problem is the use of modular robots for creating specialized robotic vehicles. A typical modular robot consists of a small number of basic building blocks that can be combined in different ways to create a robot for a specific purpose. The basic building blocks are designed with uniform docking interfaces that permit transfer of forces, moments, power, and communication between building blocks. Each building block is outfitted with a structural shell, sensors, actuators, a power source, and a payload bay. Using these basic building blocks, robots can be configured while operating to deliberately change shape by rearranging the connectivity of their building blocks to perform new missions, adapt to a changing environment, or recover from damage or failures [1-ㅡ].

For aircraft applications, this involves individual flying vehicles with the capability to attach and detach from other air vehicles during flight. Air vehicles that are composed of numerous smaller aircraft are dubbed meta aircraft, or aircraft of aircraft. The concept of meta aircraft is not new. After World War II, the concept of compound aircraft transport was investigated for its benefit in range and endurance. The project TipTow involved two F-84 aircraft linking with a larger B-29 aircraft [5] . The two F-84 aircraft would connect in flight and subsequently power down their engines. All of these tests were pilot-in-the-loop flight tests. During a flight experiment, an F-84 aircraft entered an unstable oscillation, which resulted in a catastrophic failure. The project was subsequently canceled [6]. Research has been reported on permanently connected aircraft such as the work by Moore and Maddalon [7], who considered a multibody transport aircraft consisting of two passenger jets permanently connected at the wing tips. Their analysis showed a direct decrease in weight due to lower bending moment of the connected wing as well as an $L / D$ increase of $8-10 \%$ from the single-vehicle configuration [7]. Formation flight has also been investigated due to a potential increase in $L / D$ [8]. Magill et al. considered two compound aircraft 
transport configurations where wing tips are docked permanently and another where the aircraft fly in close formation [9]. It was found that the wing tip docked configuration had a $20-40 \%$ performance benefit as compared to solo flight. Recently, Oung, et al. created the distributed flight array (DFA) [10,11]. The DFA is a set of groundbased rovers equipped with a single ducted fan and wheels to maneuver on the ground. Each rover drives independently, connects to other robots using mechanical connections, and takes off as a unit. These are unstable during solo flight [10]. Another meta aircraft is Aurora's Odysseus concept. This aircraft consists of three independent aircraft that connect in flight in a wing-tip-to-wing-tip configuration. Each aircraft is equipped with solar panels to maintain atmospheric flight indefinitely. The primary goal of this three body meta aircraft was to remain airborne and sample the wind environment continuously. Unfortunately, no simulation or prototype has been developed for this concept [12].

The work reported here explores the flight dynamics of meta aircraft configurations with a focus on the flight dynamic modes and mode shapes. The paper begins with a description of the overall flight dynamics model used for predictions and subsequently employs this model to examine different configurations and connection conditions.

\section{Meta Aircraft Simulation Model}

A meta aircraft is a discrete set of individual aircraft that are connected together in some manner to form a larger composite aircraft. Figure 1 shows two examples of different meta aircraft configurations.

The flight dynamic simulation is formed by modeling each aircraft in the meta aircraft independently. Each aircraft is excited by typical loads that an individual aircraft experiences including gravitational and aerodynamic forces and moments. The fact that aircraft in a meta aircraft configuration are connected together introduces other forces and moments that mutually excite the aircraft. Because of the close proximity of lifting surfaces on different aircraft in the meta aircraft configuration, the aerodynamic wakes from these lifting surfaces on all aircraft interact with each other. In the simulation described next, the effect is modeled using a nonlinear lifting-line approach computed as the equations of motion are numerically integrated. Also, connected aircraft create physical contact between bodies; thus, contact forces and moments between each aircraft are also present. This effect is modeled using a series of linear and rotational springs and dampers. The following subsections provide details on the overall aircraft flight dynamic model.

\section{A. Flight Dynamics}

A standard rigid body aircraft representation is used to model flight dynamic motion [13]:

$$
\left\{\begin{array}{c}
\dot{x}_{i} \\
\dot{y}_{i} \\
\dot{z}_{i}
\end{array}\right\}=\left[\begin{array}{ccc}
c_{\theta_{i}} c_{\psi_{i}} & s_{\phi_{i}} s_{\theta_{i}} s_{\psi_{i}}-c_{\phi_{i}} s_{\psi_{i}} & c_{\phi_{i}} s_{\theta_{i}} c_{\psi_{i}}+s_{\phi_{i}} s_{\psi_{i}} \\
c_{\theta_{i}} s_{\psi_{i}} & s_{\phi_{i}} s_{\theta_{i}} s_{\psi_{i}}+c_{\theta_{i}} c_{\psi \psi_{i}} & c_{\phi_{i}} s_{\theta_{i}} s_{\psi_{i}}-s_{\phi_{i}} c_{\psi_{i}} \\
-s_{\theta_{i}} & s_{\phi_{i}} c_{\theta_{i}} & c_{\phi_{i}} c_{\theta_{i}}
\end{array}\right]\left\{\begin{array}{c}
u_{i} \\
v_{i} \\
w_{i}
\end{array}\right\}
$$

$$
\begin{gathered}
\left\{\begin{array}{c}
\dot{\phi}_{i} \\
\dot{\theta}_{i} \\
\dot{\psi}_{i}
\end{array}\right\}=\left[\begin{array}{ccc}
1 & s_{\phi_{i}} t_{\theta_{i}} & c_{\phi_{i}} t_{\theta_{i}} \\
0 & c_{\phi_{i}} & -s_{\phi_{i}} \\
0 & s_{\phi_{i}} / c_{\theta_{i}} & c_{\phi_{i}} / c_{\theta_{i}}
\end{array}\right]\left\{\begin{array}{c}
p_{i} \\
q_{i} \\
r_{i}
\end{array}\right\} \\
\left\{\begin{array}{c}
\dot{u}_{i} \\
\dot{v}_{i} \\
\dot{w}_{i}
\end{array}\right\}=\frac{1}{m_{i}}\left\{\begin{array}{c}
X_{i} \\
Y_{i} \\
Z_{i}
\end{array}\right\}-S\left(\omega_{i / I}\right)\left\{\begin{array}{c}
u_{i} \\
v_{i} \\
w_{i}
\end{array}\right\} \\
\left.\left\{\begin{array}{c}
\dot{p}_{i} \\
\dot{q}_{i} \\
\dot{r}_{i}
\end{array}\right\}=\left[I_{i}\right]^{-1}\left(\left\{\begin{array}{c}
L_{i} \\
M_{i} \\
N_{i}
\end{array}\right\}-S\left(\omega_{i / I}\right)\left[I_{i}\right]\right\}\left\{\begin{array}{c}
p_{i} \\
q_{i} \\
r_{i}
\end{array}\right\}\right)
\end{gathered}
$$

Standard shorthand notation is used for trigonometric functions: $\cos (\alpha) \equiv c_{\alpha}, \sin (\alpha) \equiv s_{\alpha}$, and $\tan (\alpha) \equiv t_{\alpha}$. The skew symmetric operator is denoted by $\boldsymbol{S}()$. Multiplying this matrix by a vector is equivalent to a cross product:

$$
\boldsymbol{S}\left(\boldsymbol{\omega}_{i / I}\right)=\left[\begin{array}{ccc}
0 & -r_{i} & q_{i} \\
r_{i} & 0 & -p_{i} \\
-q_{i} & p_{i} & 0
\end{array}\right]
$$

The mass moment of inertia matrix $I_{i}$ is taken about the center of gravity of the aircraft and is positive-definite and symmetric. The applied forces and moments expressed in Eqs. (3) and (4) contain contributions from weight $(W)$, aerodynamics $(\bar{A})$, and contact $(C)$ loads. The total forces and moments applied to the aircraft expressed in the body reference frame are given next:

$$
\begin{gathered}
\left\{\begin{array}{c}
X_{i} \\
Y_{i} \\
Z_{i}
\end{array}\right\}=\left\{\begin{array}{l}
X_{W i}+X_{A i}+X_{C i} \\
Y_{W i}+Y_{A i}+Y_{C i} \\
Z_{W i}+Z_{A i}+Z_{C i}
\end{array}\right\} \\
\left\{\begin{array}{c}
L_{i} \\
M_{i} \\
N_{i}
\end{array}\right\}=\left\{\begin{array}{c}
L_{A i}+L_{C i} \\
M_{A i}+M_{C i} \\
N_{A i}+N_{C i}
\end{array}\right\}
\end{gathered}
$$

The weight contribution is given by Eq. (8):

$$
\left\{\begin{array}{c}
X_{W i} \\
Y_{W i} \\
Z_{W i}
\end{array}\right\}=m_{i} g\left\{\begin{array}{c}
-s_{\theta_{i}} \\
s_{\phi_{i}} c_{\theta_{i}} \\
c_{\phi_{i}} c_{\theta_{i}}
\end{array}\right\}
$$

The aerodynamic force for all lifting surfaces on the body (main wing, horizontal tail, and vertical tail) are calculated using welldocumented strip theory coupled to a nonlinear lifting-line wake model. All lifting surfaces are split into a discrete number of elements, and lift and drag are computed for each element. The aerodynamic forces and moments associated with the fuselage are computed using a standard aerospace expansion:

$$
\left\{\begin{array}{c}
X_{A_{i}} \\
Y_{A_{i}} \\
Z_{A_{i}}
\end{array}\right\}=\left\{\begin{array}{c}
X_{E_{i}} \\
Y_{E_{i}} \\
Z_{E_{i}}
\end{array}\right\}+\left\{\begin{array}{c}
X_{F_{i}} \\
Y_{F_{i}} \\
Z_{F_{i}}
\end{array}\right\}
$$
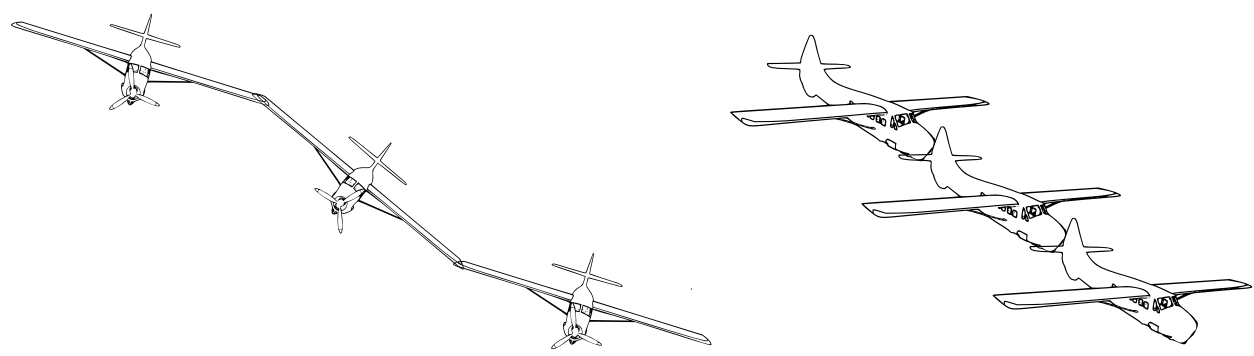

Fig. 1 Different meta aircraft configurations: wing-tip-to-wing-tip (left) and tip-to-tail (right). 
In Eq. (9), the subscripts denote contributions from all the lifting surface elements $E$ and the fuselage $F$. Equation (10) details the computation of aerodynamic forces produced by all lifting surface elements (main wing and tail surfaces). In this analysis, the horizontal and vertical tails contain one element each. Thus, the first term is summed from 0 to $N+2$, where $N$ is the number of lifting surface elements on the main wing, and the extra two terms are for the horizontal and vertical tails. Note that the matrix $\boldsymbol{T}_{j B_{i}}$ is a transformation matrix from the local $j$ th element frame to the $i$ th body aircraft frame. This allows for inclusion of sweep and dihedral effects:

$$
\left\{\begin{array}{c}
X_{E i} \\
Y_{E i} \\
Z_{E i}
\end{array}\right\}=\frac{1}{2} \rho \sum_{j=0}^{N+2} \boldsymbol{T}_{j B_{i}}^{T} S_{j} V_{j}^{2}\left\{\begin{array}{c}
C_{L j} s_{\alpha_{j}}-C_{D j} c_{\alpha_{j}} \\
0 \\
-C_{L j} c_{\alpha_{j}}-C_{D j} s_{\alpha_{j}}
\end{array}\right\}
$$

The sectional lift and drag coefficients for each element are

$$
\left\{\begin{array}{l}
C_{L j} \\
C_{D j}
\end{array}\right\}=\left\{\begin{array}{l}
C_{L 0 j}+C_{L \alpha j} \alpha_{j} \\
C_{D 0 j}+C_{D \alpha j} \alpha_{j}^{2}
\end{array}\right\}
$$

Using the aircraft velocity with respect to atmospheric winds, it is possible to compute the total velocity, angle of attack, and sideslip for each element:

$$
\begin{gathered}
V_{j}=\sqrt{u_{j}^{2}+v_{j}^{2}+w_{j}^{2}} \\
\alpha_{j}=\tan ^{-1}\left(\frac{w_{j}}{u_{j}}\right) \\
\beta_{j}=\sin ^{-1}\left(\frac{v_{j}}{V_{j}}\right)
\end{gathered}
$$

Note that these velocity components include the effects due to aircraft motion and induced velocity and are expressed in the local element frame:

$$
\left\{\begin{array}{c}
u_{j} \\
v_{j} \\
w_{j}
\end{array}\right\}=\boldsymbol{T}_{j B_{i}}\left[\left\{\begin{array}{c}
u_{i} \\
v_{i} \\
w_{i}
\end{array}\right\}+\boldsymbol{S}\left(\boldsymbol{\omega}_{i / I}\right)\left\{\begin{array}{c}
r_{x j} \\
r_{y j} \\
r_{z j}
\end{array}\right\}\right]+\left\{\begin{array}{c}
u_{I j} \\
v_{I j} \\
w_{I j}
\end{array}\right\}
$$

The previous equation contains contributions from three sources. The first is the aerodynamic velocity of the mass center of aircraft $i$. The second term is due to aircraft rotational motion, where $r_{x j}, r_{y j}$, and $r_{z j}$ are the scalar distances from the computation point of the $j$ th element to the center of mass of aircraft $i$. The final term is the induced velocity produced by all other lifting surface elements in the meta aircraft system. The fuselage is modeled using an aerodynamic expansion taken about the center of mass of the aircraft:

$$
\left\{\begin{array}{c}
X_{F i} \\
Y_{F i} \\
Z_{F i}
\end{array}\right\}=\frac{1}{2} \rho S V_{\infty}^{2}\left\{\begin{array}{c}
C_{L f} s_{\alpha}-C_{D f} c_{\alpha}+C_{x_{\delta_{t}}} \delta_{t} \\
C_{y f} \\
-C_{L f} c_{\alpha}-C_{D f} s_{\alpha}
\end{array}\right\}
$$

where the fuselage coefficients are

$$
\left\{\begin{array}{l}
C_{L f} \\
C_{D f} \\
C_{y f}
\end{array}\right\}=\left\{\begin{array}{c}
C_{L 0 f}+C_{L \alpha f} \alpha+\frac{c}{2 V_{\infty}} C_{L q f} q+C_{L \delta_{e}} \delta_{e} \\
C_{D 0 f}+C_{D \alpha f} \alpha^{2} \\
C_{y \beta f} \beta+C_{y \delta_{r}} \delta_{r}+\frac{b}{2 V_{\infty}}\left(C_{y p f} p+C_{y r f} r\right)
\end{array}\right\}
$$

The body aerodynamic moment is then computed by moments from all lifting surfaces as well as including the effect of forces offset from the center of mass.

$$
\begin{aligned}
& \left\{\begin{array}{c}
L_{A i} \\
M_{A i} \\
N_{A i}
\end{array}\right\}=\frac{1}{2} \rho \sum_{j=0}^{N+2}\left(\boldsymbol{T}_{j B_{i}}^{T} V_{j}^{2} S_{j} c_{j}\left\{\begin{array}{c}
0 \\
C_{m j} \\
0
\end{array}\right\}+\boldsymbol{S}\left(\boldsymbol{r}_{i \rightarrow j}\right)\left\{\begin{array}{c}
X_{A j} \\
Y_{A j} \\
Z_{A j}
\end{array}\right\}\right) \\
& +\frac{1}{2} \rho S V_{\infty}^{2}\left\{\begin{array}{c}
C_{l f} \\
C_{m f} \\
C_{n f}
\end{array}\right\}
\end{aligned}
$$

The fuselage aerodynamic moment coefficients are given by

$$
\left\{\begin{array}{c}
C_{l f} \\
C_{m f} \\
C_{n f}
\end{array}\right\}=\left\{\begin{array}{c}
C_{l \beta f} \beta+\frac{b}{2 V_{\infty}}\left(C_{l p f} p+C_{l r f} r\right)+C_{l \delta_{a}} \delta_{a}+C_{l \delta_{r}} \delta_{r} \\
C_{m 0 f}+C_{m \alpha f} \alpha+\frac{c}{2 V_{\infty}} C_{m q f} q+C_{m \delta_{e}} \delta_{e} \\
\frac{b}{2 V_{\infty}}\left(C_{n p f} p+C_{n r f} r\right)+C_{n \beta f} \beta+C_{n \delta_{a}} \delta_{a}+C_{n \delta_{r}} \delta_{r}
\end{array}\right\}
$$

The aerodynamic coefficients in Eqs. $(\underline{10}, 11,16-19)$ can be obtained from flight data, aerodynamic modeling, and wind-tunnel tests [14].

\section{B. Nonlinear Lifting-Line Wake Model}

Trailing vortices from different lifting surfaces on the same aircraft or different aircraft connected together interact with each other causing a change in aerodynamic loading. Figure 2 shows an example three aircraft configuration. Each dot is an aerodynamic element containing three vortices, one bound vortex, and two trailing vortices. All the vortices shown in this figure interact with each other, changing the overall lift distribution of the system.

To compute this interaction, the aerodynamic model uses a nonlinear lifting-line wake model for an incompressible and inviscid flowfield about a finite wing [6,15-18]. Each lifting surface element contains one horseshoe vortex where the strength of the vortex is unknown. The method employed here is an iterative method where an initial induced velocity is assumed at each element, and Eq. (15) is used to compute the local velocity and angle of attack at each element. Then, Eq. (11) is used to compute the lift coefficient at each element. Using the Kutta-Joukowski theorem, the lift coefficient is shown to be a function of the strength of the horseshoe vortex at the $j$ th element

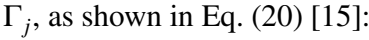

$$
\Gamma_{j}=C_{L j} c_{j} V_{j} / 2
$$

Using the previous equation, the induced velocity contribution of every element is computed. The Biot-Savart law is used to calculate the induced velocity of a vortex filament and is used to generate Eq. (미) $[\underline{6}, \underline{15}, \underline{16}]$ :

$$
W_{A B, C}=\frac{\Gamma_{j}}{4 \pi r_{p}}\left[\cos \left(\gamma_{1}\right)-\cos \left(\gamma_{2}\right)\right]
$$

where $W_{A B, C}$ is the downwash velocity at point $\mathrm{C}$ in the local element frame caused by the vortex filament from point $A$ to point $B$ and $\gamma_{1}$ and $\gamma_{2}$ are the angles between the left and right trailing vortices and point $C$. Note, however, that the induced velocity of the $j$ th element is equal to the sum of the induced velocities of all other horseshoe vortices in this multi-element system. Thus, the induced velocity $W_{I, j}$ of the $j$ th element can be given in matrix form by the next equation:

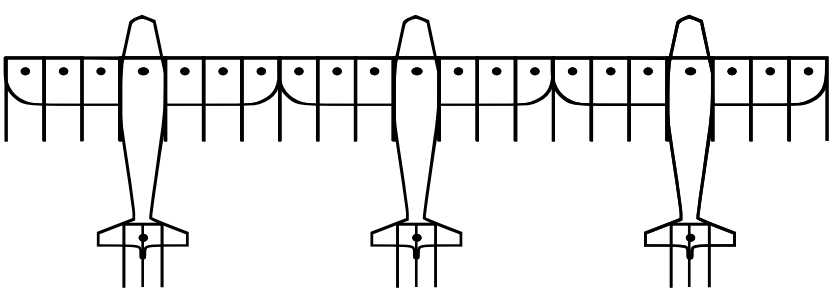

Fig. 2 Aerodynamic wake interaction in a three-aircraft wing-tip-towing-tip configuration. 


$$
\left\{\begin{array}{c}
W_{I, 1} \\
W_{I, 2} \\
\vdots \\
W_{I, N+2}
\end{array}\right\}=\left[\begin{array}{cccc}
C_{1,1} & C_{1,2} & \ldots & C_{1, N+2} \\
C_{2,1} & C_{2,2} & \ldots & C_{2, N+2} \\
\ldots & \ldots & \ddots & \ldots \\
C_{N+2,1} & C_{N+2,2} & \ldots & C_{N+2, N+2}
\end{array}\right]\left\{\begin{array}{c}
\Gamma_{1} \\
\Gamma_{2} \\
\vdots \\
\Gamma_{N+2}
\end{array}\right\}
$$

In this equation, the matrix $C_{j, k}$ relates the strength of the $j$ th horseshoe vortex on the $k$ th element. An iterative method is used to solve for the induced velocity using Eqs. (15), (11), (20), and (22) in that respective order. The numerical computation of the wake model is iterated using this method at each time step until the difference between the norm of the previous solution and the norm of the current solution is less than $1 \mathrm{e}-10$. This usually requires about 10 iterations to converge between integration time steps. This method has been validated against analytical solutions with good agreement $[15,16]$.

\section{Contact Model}

Contact forces and moments exist between connected bodies at the joint connection point. Because aircraft are permanently connected, the simulation models the connection mechanism with translational and rotational springs and dampers given by the next equations:

$$
\begin{aligned}
& \left\{\begin{array}{c}
X_{C i} \\
Y_{C i} \\
Z_{C i}
\end{array}\right\}=\left\{\begin{array}{c}
K_{x} \Delta x_{W i j}+C_{x} \Delta \dot{x}_{W i j} \\
K_{y} \Delta y_{W i j}+C_{y} \Delta \dot{y}_{W i j} \\
K_{z} \Delta z_{W i j}+C_{z} \Delta \dot{z}_{W i j}
\end{array}\right\} \\
& \left\{\begin{array}{c}
L_{C i} \\
M_{C i} \\
N_{C i}
\end{array}\right\}=\left\{\begin{array}{c}
K_{\phi} \Delta \phi_{i j}+C_{\phi} \Delta \dot{\phi}_{i j} \\
K_{\theta} \Delta \theta_{i j}+C_{\theta} \Delta \dot{\theta}_{i j} \\
K_{\psi} \Delta \psi_{i j}+C_{\psi} \Delta \dot{\psi}_{i j}
\end{array}\right\}
\end{aligned}
$$

where $\Delta x_{W i j}, \Delta y_{W i j}$, and $\Delta z_{W i j}$ are the inertial distances between the wing tip of the $i$ th and $j$ th aircraft. The values $\Delta \phi_{i j}, \Delta \theta_{i j}$, and $\Delta \psi_{i j}$ are the Euler angle differences between the $i$ th and $j$ th aircraft.

\section{Aircraft Description}

To explore the flight dynamics of meta aircraft, an example small aircraft is used for all simulation results. The example aircraft uses the aerodynamics and mass properties of a single-propeller, high-wing conventional aircraft as reported by [14]. The mass of the nominal single aircraft is $5.6 \mathrm{~kg}$. The mass moments of inertia are $I x x=$ $0.4923 \mathrm{~kg} \cdot \mathrm{m}^{2}, I y y=0.5111 \mathrm{~kg} \cdot \mathrm{m}^{2}$, and $I z z=0.8470 \mathrm{~kg} \cdot \mathrm{m}^{2}$. The main wing has a $2.04 \mathrm{~m}$ span and a $0.3215 \mathrm{~m}$ chord with a cambered airfoil (NACA 1412); thus, the maximum thickness is $t=0.0386 \mathrm{~m}$. The lift coefficient at zero angle of attack is 0.062 , and the lift slope is $5.195 / \mathrm{rad}$. The horizontal tail is $0.72 \mathrm{~m}$ in span with a $0.295 \mathrm{~m}$ chord. The vertical tail has a half-span of $0.36 \mathrm{~m}$ and a chord of $0.25 \mathrm{~m}$. Both horizontal and vertical tails use symmetric airfoils and are positioned $1.4 \mathrm{~m}$ from the center of mass of the aircraft. The aircraft uses a conventional control layout with an elevator to control pitch, ailerons to control roll angle, rudder to control sideslip, and a propeller for thrust. This aircraft has a nominal flight speed of $20 \mathrm{~m} / \mathrm{s}$.

Connection joint elastic properties are set with stiff linear elastic elements and relatively soft rotational elastic elements to model connection mechanisms that allow relative rotation. Thus, the linear springs and dampers have been set to $10,000 \mathrm{~N} / \mathrm{m}$ and $40 \mathrm{~N} \cdot \mathrm{s} / \mathrm{m}$ respectively. The rotational springs and dampers have been set to allow relative rotation between each aircraft. The values of the roll, pitch, and yaw stiffness are $370 \mathrm{~N} \cdot \mathrm{m} / \mathrm{rad}, 2580 \mathrm{~N} \cdot \mathrm{m} / \mathrm{rad}$, and $2580 \mathrm{~N} \cdot \mathrm{m} /$ rad, repectively. The roll, pitch, and yaw damping are $1.5 \mathrm{~N} \cdot \mathrm{m} \cdot \mathrm{s} /$ $\mathrm{rad}, 10 \mathrm{~N} \cdot \mathrm{m} \cdot \mathrm{s} / \mathrm{rad}$, and $10 \mathrm{~N} \cdot \mathrm{m} \cdot \mathrm{s} / \mathrm{rad}$, respectively.

The fuselage coefficients are obtained using a numerical fitting procedure so that the forces and moments due to all lifting surfaces and the fuselage are equal to the forces and moments produced by the full aerodynamic expansion given by [14]. The results presented in this section were done for three, five, and seven elements on the main wing, and no significant difference was found. Note that propeller aerodynamic wash effects are not modeled.

\section{Flight Dynamic Modes of Motion for Meta Aircraft}

To obtain the flight dynamic modes of motion of the meta aircraft system, the trim state of a connected meta aircraft configuration is obtained through adjustment of elevator inputs to track a prescribed altitude, thrust inputs to track a desired flight speed, aileron inputs to balance roll moments, and rudder commands to balance yaw moments. Aircraft control inputs are adjusted while integrating the equations of motion until the system reaches steady state with all state derivatives (except $\dot{x}_{i}$ ) less than $1 \mathrm{e}-8$. This trimming procedure was found to work robustly for all configurations examined.

Once the trim state of a configuration is found, a linear timeinvariant model is obtained numerically using forward finite differencing to compute the Jacobian of the nonlinear model [19]. Each state is perturbed from trim by $1 \mathrm{e}-6$ to compute numerical derivatives. A $12 N_{A C}$ state linear time-invariant dynamic model results with $12 N_{A C}$ associated eigenvalues (modes) and eigenvectors (mode shapes). For four aircraft composing a meta aircraft configuration, the linear model has 48 states. For a body in free flight, 12 of these modes and modes shapes are associated with rigid aircraft flight dynamic motion of the entire meta aircraft configuration. These are relatively low frequency and are associated with the global flight dynamic behavior of the aircraft. They involve the classical phugoid, short period, dutch roll, spiral, and roll modes [13,19]. The remaining modes in the linear time-invariant model are associated with the flexible modes of motion. The flight dynamic modes of a single aircraft are shown in Fig. 3 . Specifically, the short period mode is $(-5.92 \pm 9.22 i)$, the phugoid mode is $(-0.032 \pm 0.61 i)$, the roll mode is $(-16.93)$, the dutch roll mode is $(-0.42 \pm 2.31 i)$, and the spiral mode is $(-0.029)$. The same analysis is conducted for aircraft connected tip-to-tail and wing-tip-to-wing-tip. To establish a pattern, five aircraft are examined for both wing-tip-to-wing-tip and tip-to-tail connected flight.

To understand the physical and aerodynamic changes when aircraft are connected together, the results from the nonlinear model are compared to analytical expressions by Phillips [19]. It is possible to obtain analytic expressions for each flight dynamic mode; however, these analytic expressions require the full aerodynamic expansion of an aircraft rather than the split aerodynamic model used in this formulation. To use these expressions, the meta aircraft is assumed to act as a rigid body, and equivalent aerodynamic coefficients are obtained. Equations can be created for all longitudinal and lateral coefficients and used in approximate flight dynamic expressions [19]. It is assumed that the geometry of the meta aircraft can be scaled, such that the planform area of the entire system $S_{T}=N_{A C} S$. Similarly, for wing-tip-to-wing-tip connected flight, the wingspan is $b_{T}=N_{A C} b$; however, the chord is kept constant. In tip-

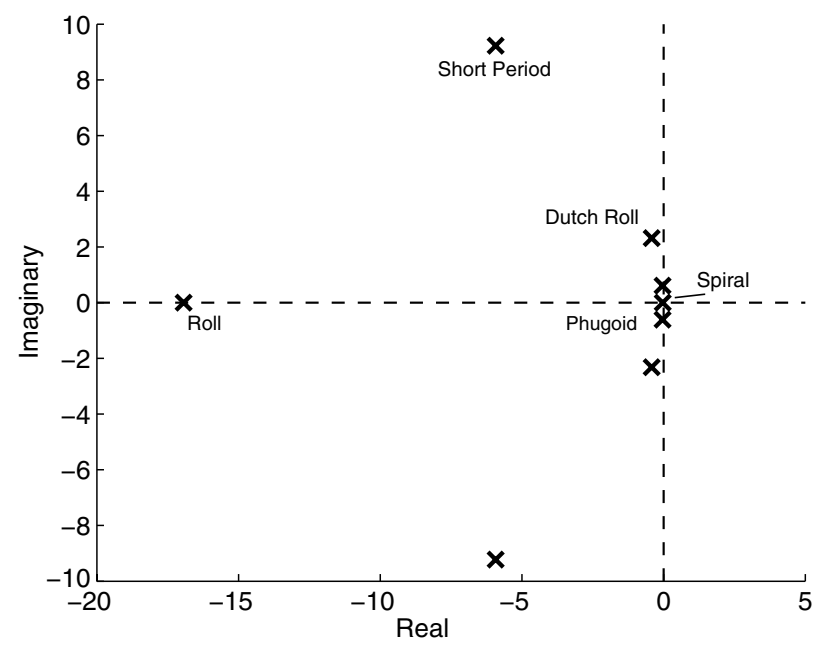

Fig. 3 Single-aircraft flight dynamic modes. 


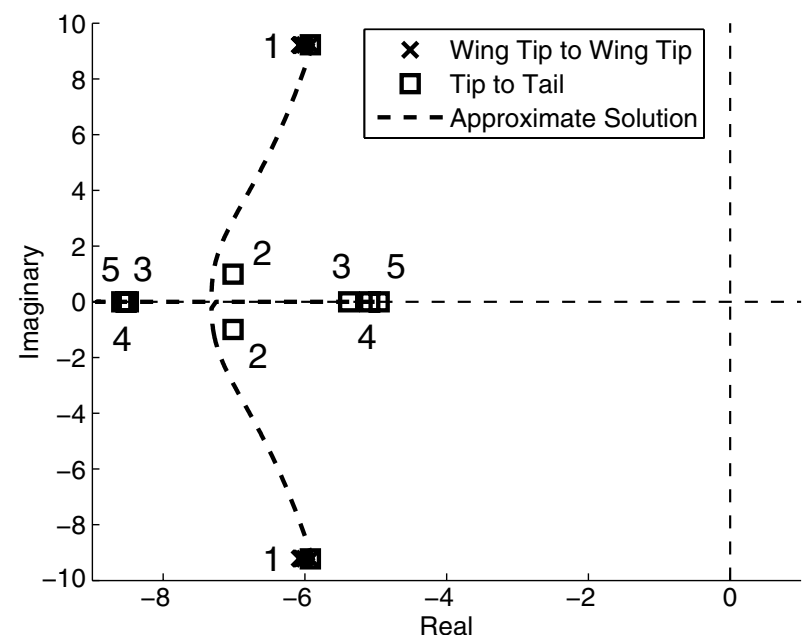

Fig. 4 Short period mode as a function of number of connected aircraft.

to-tail connected flight, $c_{T}=N_{A C} c$ and the span is kept constant. The pitch, roll, and yaw inertias are computed using the parallel axis theorem. It is easy to see that, in wing-tip-to-wing-tip connected flight, the pitch inertia increases linearly, whereas the roll and yaw inertias increase quadratically. Conversely, in tip-to-tail connected flight, the roll inertia increases linearly with number of connected aircraft, and the yaw and pitch inertias increase quadratically. Using the equations for geometry, mass, inertia, and equivalencing the overall aerodynamic coefficients, analytical expressions in [19] are used to compute the flight dynamic modes. The results of these equations are plotted alongside the numerical results for wing-tip-towing-tip connected flight and tip-to-tail connected flight. In addition to simply plotting the discrete points from single to multiple connected aircraft, it is possible to interpolate all coefficients in between discrete solutions and obtain a smooth approximation for each mode.

Figure 4 shows the change in the short period mode. Each discrete point is the addition of an extra aircraft, as shown by the number above each pole. For example, " 3 " is associated with three aircraft connected either wing-tip-to-wing-tip or tip-to-tail. The short period mode is a relatively fast mode that can be excited by a step change in the elevator. The aircraft exhibits a quick oscillation about the pitch axis, which quickly damps out. The short period mode is largely unchanged for wing-tip-to-wing-tip connected flight, which is expected because this is a pure longitudinal mode. However, the short period mode changes drastically for tip-to-tail connected flight. This mode becomes critically damped once there are three or more aircraft connected together. Figure 5 indicates a similar change in the phugoid mode. Just as in the short period mode, a negligible change is seen for wing-tip-to-wing-tip flight versus a drastic change in tip-totail flight. The underlying physical phenomenon can be explained by

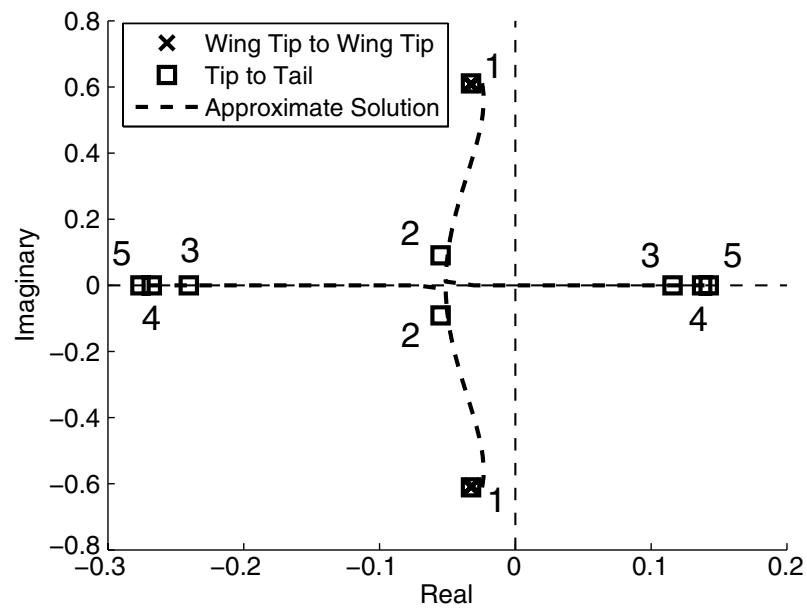

Fig. 5 Phugoid mode as a function of number of connected aircraft. examining the change in geometry and aerodynamics. In wing-tip-towing-tip connected flight, the pitch inertia of the aircraft increase linearly as well as the overall planform area. In addition, when the aircraft pitches about the $y$ axis, it is a symmetric pitch, that is the overall pitch rate of the entire meta aircraft is equal to the pitch rate of each individual aircraft. In addition, the angle of attack of each aircraft is identical. These effects cause all meta aircraft longitudinal coefficients to be constant with respect to the number of connected aircraft, resulting in nearly identical short period and phugoid modes. The slight difference is caused by the small change in trim state and trim controls when multiple aircraft are connected.

The tip-to-tail configuration has a different aerodynamic and geometric change. First, the pitch inertia increases quadratically. Aerodynamically, an overall pitch rate on the meta aircraft results in a nonuniform angle of attack on all aircraft, that is a positive pitch rate leads to an increase in angle of attack on trailing aircraft and a decrease in angle of attack on leading aircraft. This effect causes the pitch damping coefficient to change. This change in pitch damping, coupled with the increase in pitch inertia, causes the longitudinal modes to become critically damped after two aircraft. In addition to becoming critically damped, the phugoid mode becomes unstable. Because this is a mode along the pitch axis, this mode can be seen as a slow and unstable increase in pitch. Tip-to-tail connected flight has an axis of symmetry along the roll axis, resulting in a nearly constant roll mode as a function of the number of connected aircraft. However, because the trailing aircraft are flying in the wake of the leading aircraft, the roll damping coefficient actually drops slightly, resulting in a drop in the roll mode for tip-to-tail flight as depicted in Fig. 6.

Wing-tip-to-wing-tip connected flight creates an increase in roll damping due to the aerodynamic center offset of each aircraft from the center of mass. However, because the roll inertia of the wing-tipto-wing-tip meta aircraft increases quadratically, the roll mode frequency decreases. After about two to three connected aircraft, these two effects cancel each other out, and the roll mode stays constant. The remaining two modes involve sideslipping, rolling, and yawing motion. As such, these modes exhibit some longitudinal and lateral dynamic coupling, which causes these modes to change for both wing-tip-to-wing-tip and tip-to-tail connected flight. Figure 7 depicts the change in the dutch roll mode.

The tip-to-tail configuration causes this mode to become critically damped similar to the phugoid mode. The underlying physical phenomena is mainly caused by the increase in the magnitude of the yaw damping coefficient. Notice that the phugoid mode becomes critically damped for tip-to-tail connected flight due to a similar increase in the pitch damping coefficient. Similarly, the yaw damping coefficient increases, causing the dutch roll mode to become critically damped. In addition, the yaw and pitch moments of inertia increase quadratically with the number of connected aircraft. These two effects lead to critically damped dutch roll and longitudinal modes. Wing-tip-to-wing-tip connected flight does not exhibit a large

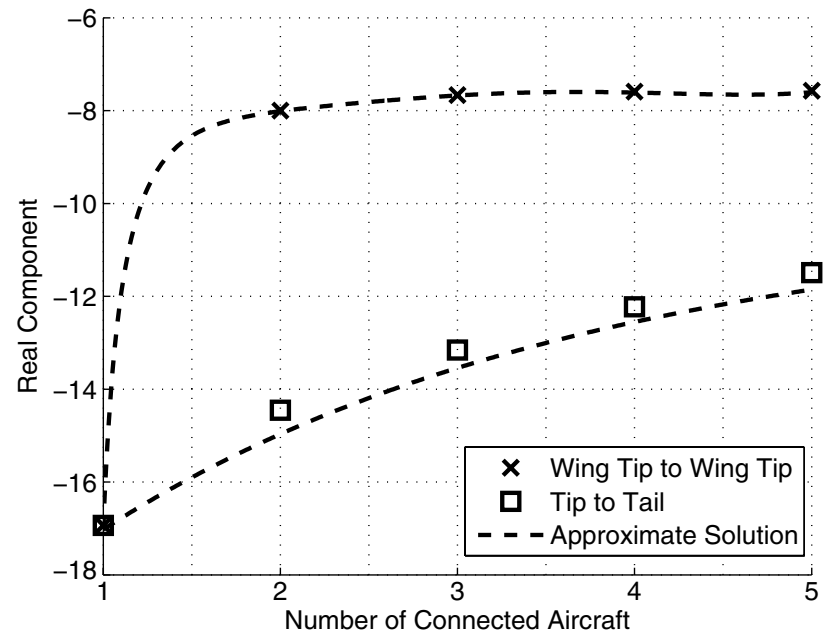

Fig. 6 Roll mode as a function of number of connected aircraft. 


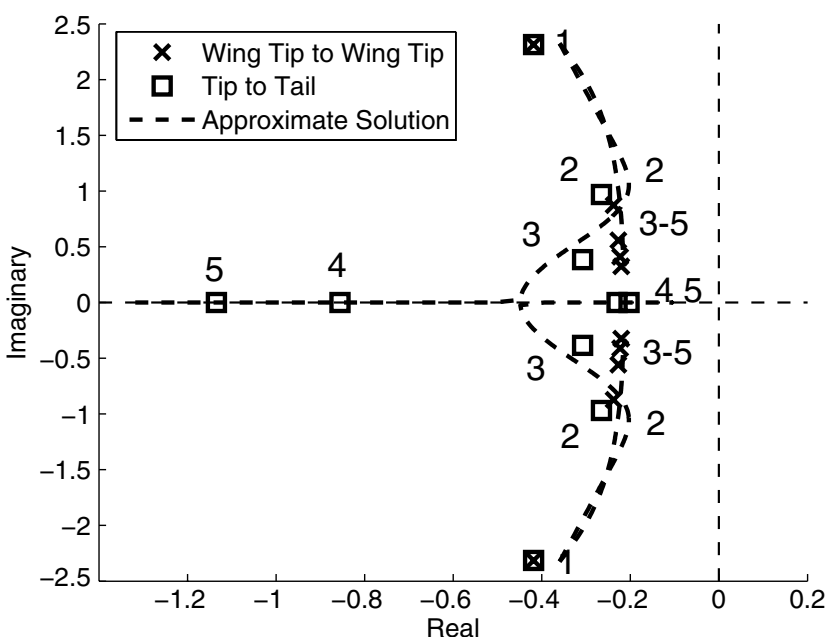

Fig. 7 Dutch roll mode as a function of number of connected aircraft.

increase in yaw damping but rather an increase in roll damping. This increase in roll damping coupled with an increase in the roll and yaw moments of inertia lead to increased damping in the dutch roll mode.

The final lateral mode, the spiral mode, changes most significantly for tip-to-tail connected flight as shown in Fig. 8. The mode becomes more convergent for two aircraft and then becomes more unstable with each added aircraft. Wing-tip-to-wing-tip connected flight, however, has a smooth increase in the mode moving from -0.0288 for the single aircraft and 0.0282 for five connected aircraft.

The change in this mode can be explained by examining the approximate solution for the spiral mode given by Eq. (24) [19]:

$$
\lambda=-\frac{g}{V_{T}} \frac{C_{L \beta_{T}} C_{N R_{T}}-C_{L R_{T}} C_{N \beta_{T}}}{C_{L \beta_{T}} C_{N P_{T}}-C_{L P_{T}} C_{N \beta_{T}}}
$$

In this equation, the total meta aircraft velocity $V_{T}$ is very insensitive to the number of connected aircraft. This mode is thus affected by the roll and yaw coefficients due to sideslipping, rolling, and yawing. It has already been discussed that the roll damping coefficient increases significantly for wing-tip-to-wing-tip connected flight and decreases slightly for tip-to-tail connected flight. In addition, the yaw damping coefficient increases in magnitude for tip-to-tail connected flight while remaining largely constant for wing-tip-to-wing-tip connected flight. The roll moment due to sideslip decreases slightly for wingtip-to-wing-tip connected flight but remains negative. This coefficient, however, changes sign for tip-to-tail connected flight again due to the trailing aircraft flying in the wake of the leading aircraft. All of these effects coupled with similar changes in other lateral coefficients leads to this change in the spiral mode. Table 1 contains a

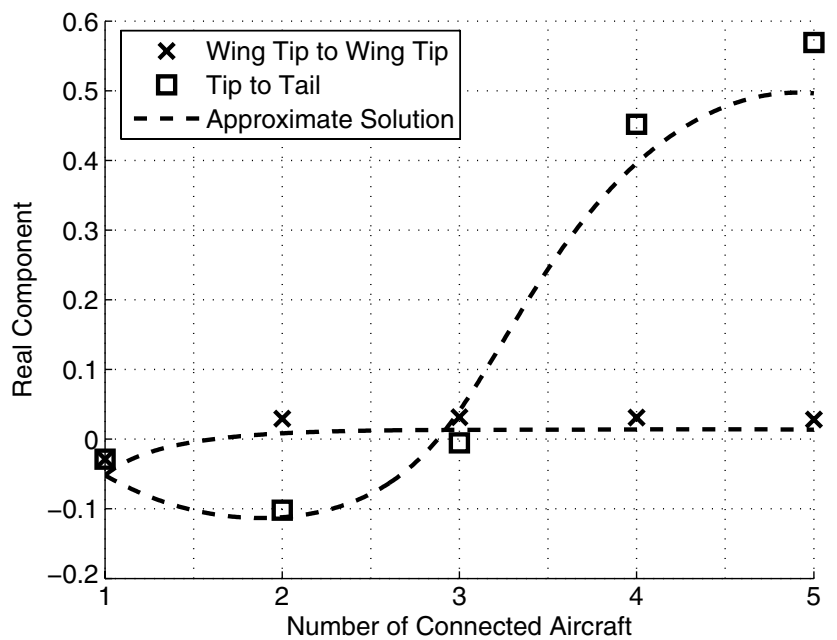

Fig. 8 Spiral mode as a function of number of connected aircraft.

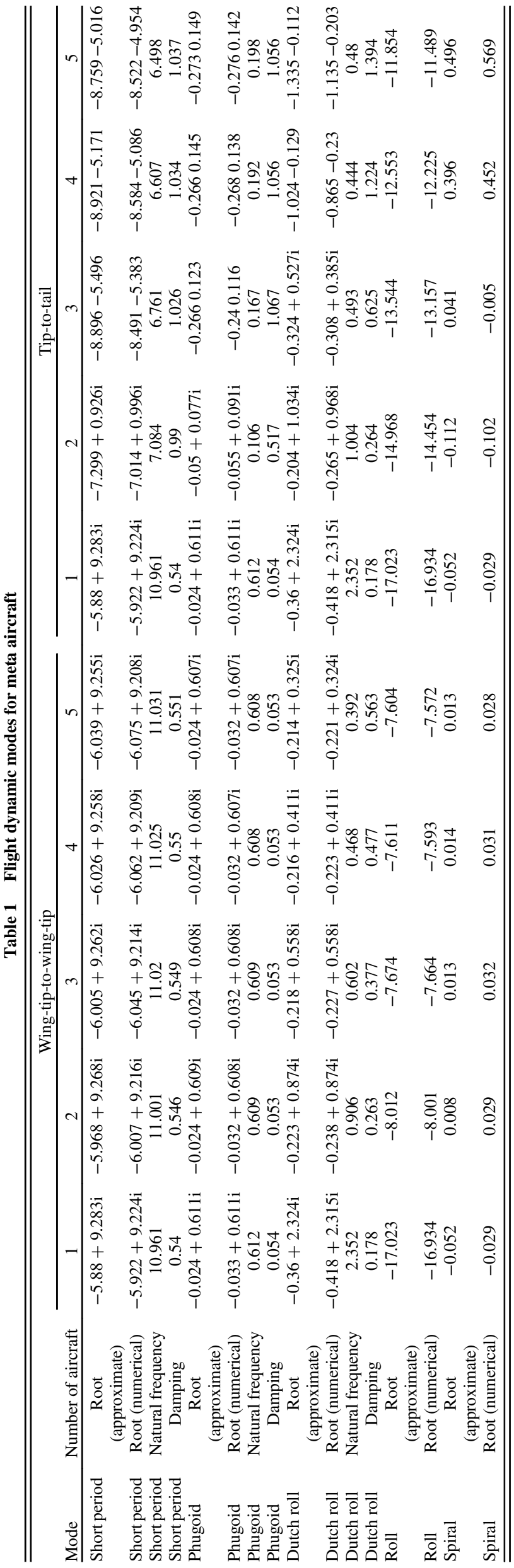




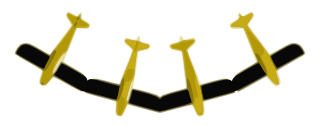

$-0.6+15.7 i$
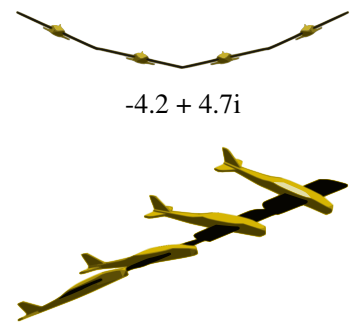

$-8.2+54.6 \mathrm{i}$
Lead Lag Modes

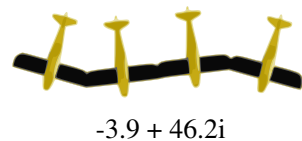

Flapping Modes

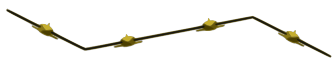

$-6.0+19.9 i$

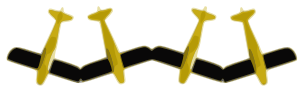

$-18.2+93.5 i$

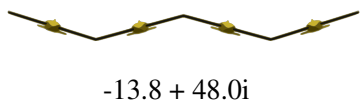

Twisting (Pitch) Modes

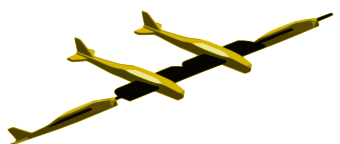

$-22.5+98.4 \mathrm{i}$

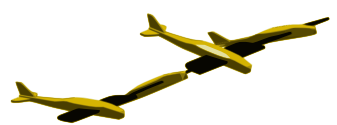

$-36.7+126.5 i$

Fig. 9 Flexible wing-tip-to-wing-tip modes.

comprehensive list of all flight dynamic modes as a function of connected aircraft along with the approximate solution from Phillips [19].

\section{Flexible Modes of Motion for Meta Aircraft}

In addition to changing the flight dynamic modes of motion, meta aircraft systems also contain flexible modes. These flexible modes are a direct result of relative motion at the joints that connect aircraft. For the four-aircraft system, the linear time-invariant model contains 48 states. Section IV described the 12 flight dynamic modes of motion. The remaining 36 modes are associated with translational flexible modes (18 eigenvalues) and rotational flexible modes (18 eigenvalues). This section deals with the modes associated with relative rolling, pitching, and yawing between each connected aircraft, resulting in $3\left(N_{A C}-1\right)$ modes with $6\left(N_{A C}-1\right)$ eigenvalues. Motion along each axis is given a name based on the axis of rotation. For wing-tip-to-wing-tip connected flight, roll motion is called flapping, pitch motion is called the twisting (pitch) mode, and yaw motion is called the lead-lag mode. For tip-to-tail connected flight, roll motion is called the twisting (roll) mode, whereas pitch and yaw motion are called porpoising and snaking modes. Figures 9 and 10 show the flexible modes of wing-tip-to-wing-tip flight and tip-totail flight separated by motion along the yaw plane, pitch plane, and roll plane for four aircraft.

The numbers below each graphic show the eigenvalue of each mode. As mentioned previously, the number of flexible rotational modes is equal to $3\left(N_{A C}-1\right)$. For four connected aircraft, there are a total of nine rotational flexible modes, with three modes along each rotational axis. For each configuration, the rows represent each axis of relative motion, and the columns are split into the lowest and highest natural frequencies. The highest-frequency mode is charac- terized by alternating angles between each aircraft. For example, in Fig. 9, the fastest lead-lag mode is characterized by the first aircraft having a positive yaw angle and the second having a negative yaw angle. This pattern is repeated for the next two aircraft. Each aircraft is 180 degrees out of phase with the aircraft to its left and to its right. Similarly, the flapping modes alternate their roll angles, and the twisting (pitch) modes alternate their pitch angles. The middle column is characterized by the two outside aircraft oscillating in phase, whereas the other two middle aircraft oscillate 180 degrees out of phase. The low-frequency flexible mode places an axis of symmetry between the second and third aircraft. Aircraft one and two oscillate in phase with varying magnitudes, whereas aircraft three and four oscillate out of phase with aircraft one and two.

\section{Effect of Aircraft Connection Characteristics}

The frequency and damping of the flexible modes are altered by changing the joint characteristics. For example, if stiffness is added to the roll axis of the joint for the wing-tip-to-wing-tip configuration, the flapping modes will have higher frequencies. To highlight the changes to the flexible modes, the rotational springs and dampers are varied for two aircraft connected wing-tip-to-wing-tip, and root locus plots are created to show the change in lead-lag, twist (pitch), and flapping modes. Because there are only two aircraft connected, the number of flexible modes is 3 , which makes the analysis much simpler. The nominal value of the rotational springs are 370, 2580, and $2580 \mathrm{~N} \cdot \mathrm{m} / \mathrm{rad}$ along the roll, pitch, and yaw axes, respectively. The rotational dampers are $1.5,10$, and $10 \mathrm{~N} \cdot \mathrm{m} \cdot \mathrm{s} / \mathrm{rad}$ along the roll, pitch, and yaw axes, respectively. The linear springs and dampers are held constant throughout this section at $10,000 \mathrm{~N} / \mathrm{m}$ and $40 \mathrm{~N} \cdot \mathrm{s} / \mathrm{m}$, respectively. Using these nominal values of springs and dampers, the nominal values of the three flexible modes are a
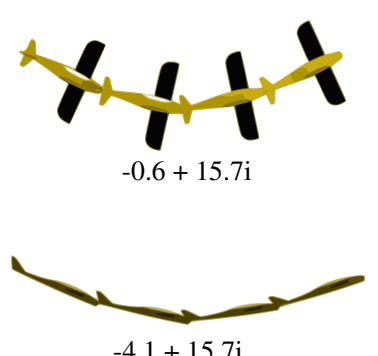

$-4.1+15.7 i$

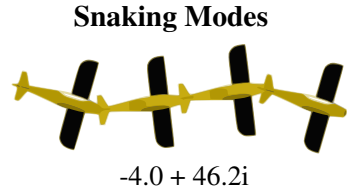

Porpoising Modes

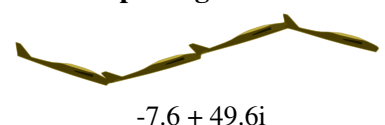

Twisting (Roll) Modes

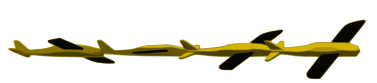

$-9.2+20.3 \mathrm{i}$

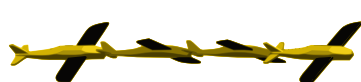

$-12.6+38.9 i$
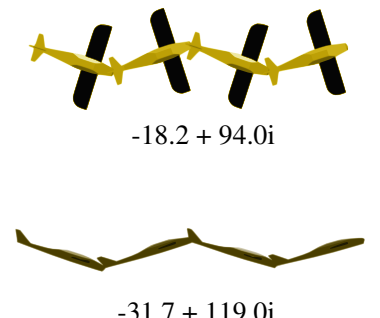

$-18.2+94.0 \mathrm{i}$

$-31.7+119.0$

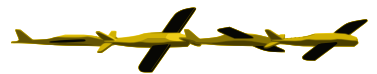

$-15.2+51.0 \mathrm{i}$

Fig. 10 Flexible tip-to-tail modes. 


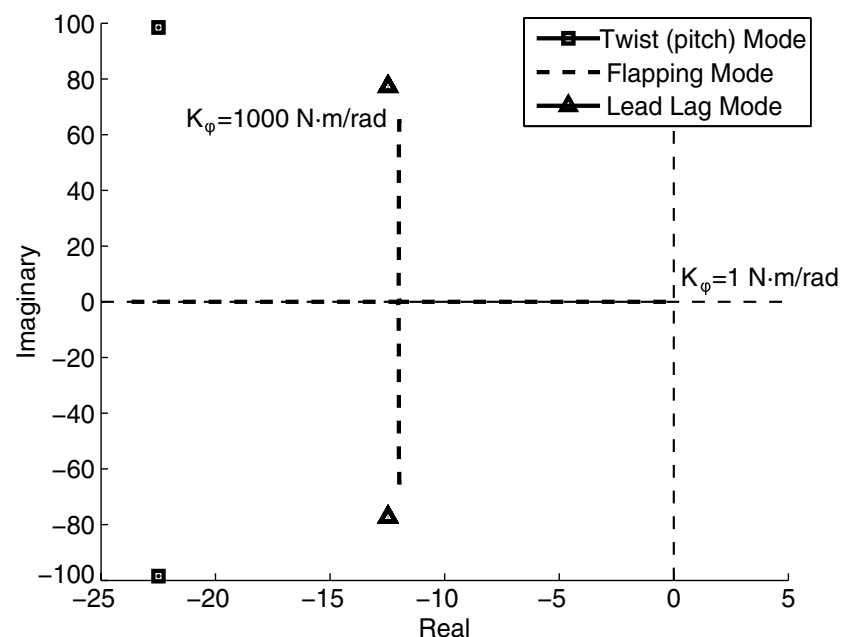

Fig. 11 Change in flexible modes as a function of roll stiffness $K_{\phi}$.

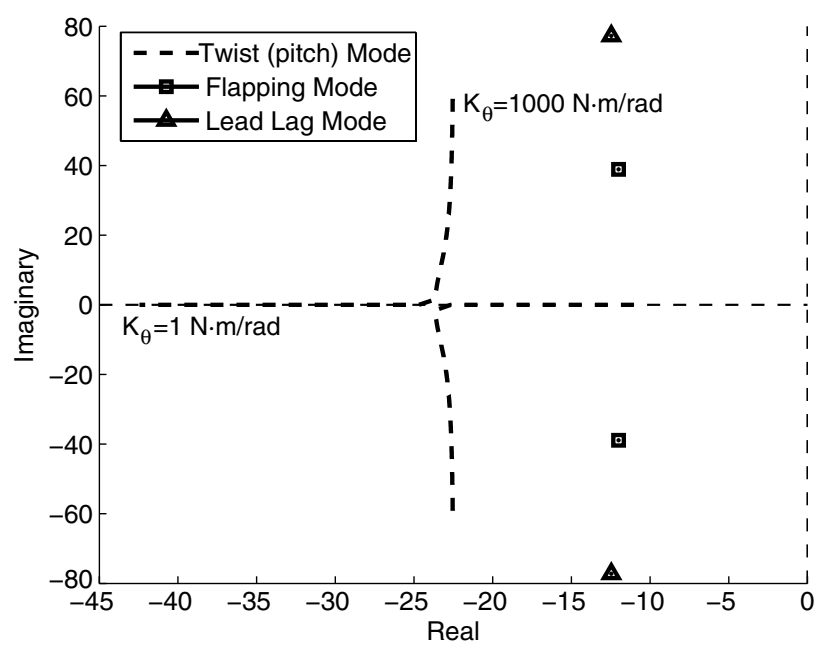

Fig. 12 Change in flexible modes as a function of pitch stiffness $K_{\theta}$.

flapping mode of $(-12.0 \pm 38.8)$, a twist (pitch) mode of $(-22.5 \pm 98.5 i)$, and a lead-lag mode of $(-12.5 \pm 77.2 i)$.

Figure 11 shows a root locus of all three flexible modes, where $K_{\phi}$ is varied from 1 to $1000 \mathrm{~N} \cdot \mathrm{m} / \mathrm{rad}$. Increasing $K_{\phi}$ is analogous to adding stiffness to the aircraft joint along the roll axis such that the aircraft resists rolling and acts to keep the roll angles of each aircraft the same. As such, the flapping mode moves from a critically damped mode to an oscillatory mode with two complex conjugate pairs. In

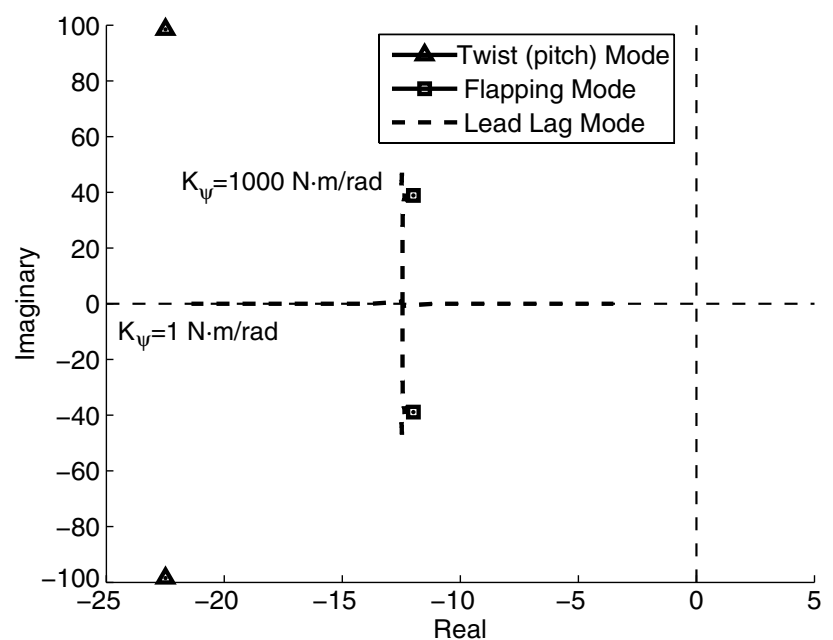

Fig. 13 Change in flexible modes as a function of yaw stiffness $K_{\psi}$.

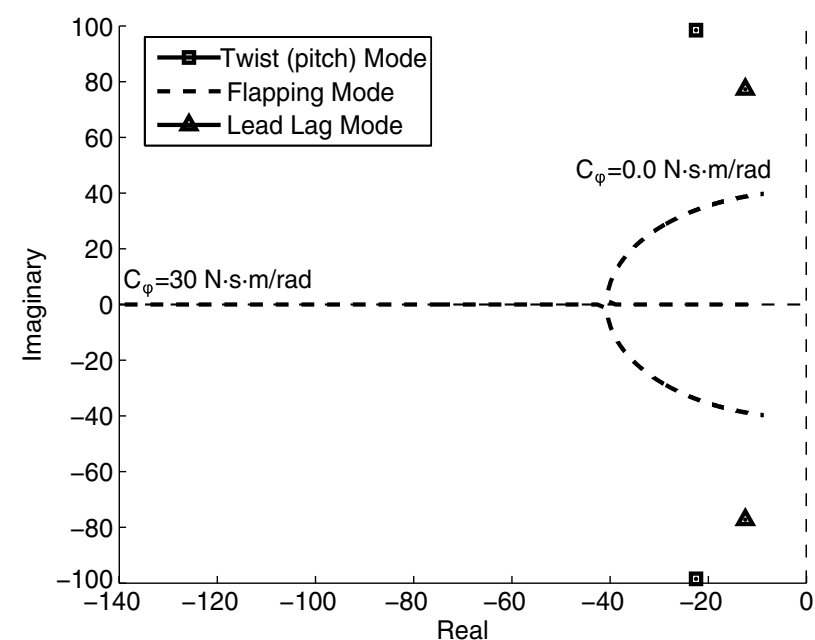

Fig. 14 Change in flexible modes as a function of roll damping $C_{\phi}$.

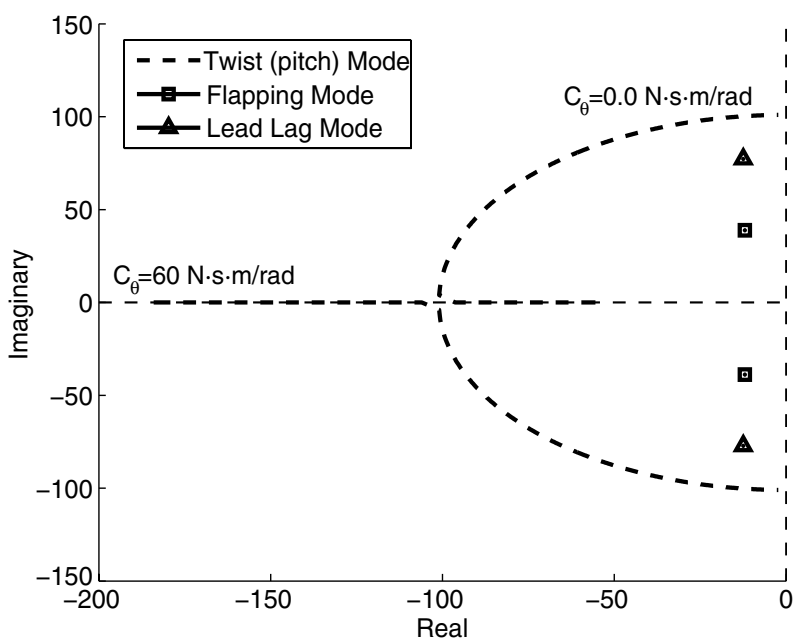

Fig. 15 Change in flexible modes as a function of pitch damping $C_{\theta}$.

addition, adding stiffness to the roll axis has no significant effect on the twist (pitch) and lead-lag modes, highlighting the decoupled nature of these three flexible modes. In addition, this study shows that, if a different flapping frequency is desired, the designer merely must change the roll stiffness of the connection joint.

A similar result can be seen when varying the rotational stiffness about the pitch and yaw axes $\left(K_{\theta}, K_{\psi}\right)$. Figures 12 and 13 show the effect of varying these two spring constants. When varying $K_{\theta}$, the

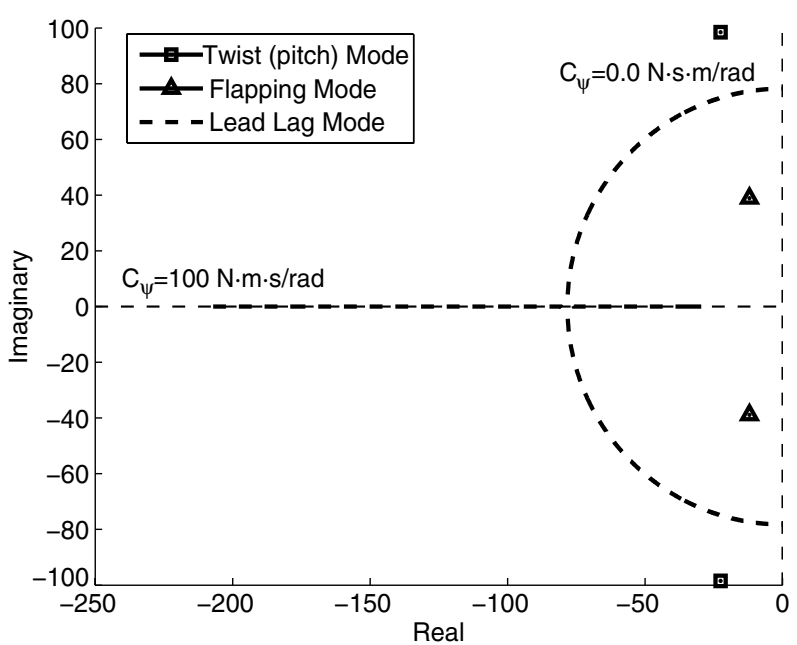

Fig. 16 Change in flexible modes as a function of yaw damping $C_{\psi}$. 
flapping and lead-lag modes remain at their nominal values while the twist (pitch) mode changes significantly. When varying $K_{\psi}$, the twist (pitch) and flapping modes remain at their nominal values while the lead-lag mode changes significantly. At a certain value of rotational stiffness, the mode switches from a critically damped mode to an oscillatory mode.

Similar effects can been seen when varying the damping coefficients along all three axes. Figures 14-16 show the root locus plots when varying the rotational damping coefficients $C_{\phi}, C_{\theta}$, and $C_{\psi}$. All flexible modes can be switched from critically damped modes to oscillatory modes by merely changing the damping coefficients at the joint.

\section{Conclusions}

These fundamental dynamics of meta aircraft are different from the basic individual aircraft kernel used to construct the meta aircraft configuration. This has practical implications for control law design of meta aircraft configurations where aircraft can attach and detach in an arbitrary fashion. For wing-tip-to-wing-tip connected flight, the flight dynamic longitudinal modes are unaffected, whereas the lateral modes change considerably. This is due to the pitch axis symmetry of wing-tip-to-wing-tip connected flight. The roll mode, however, declines due to the quadratic increase in roll inertia and the change in roll damping. For tip-to-tail flight, it is found that the longitudinal modes become overdamped after three connected aircraft and can even become unstable. The pitch axis symmetry that was encountered in wing-tip-to-wing-tip connected flight is seen as a roll axis symmetry in tip-to-tail connected flight. The roll mode for tip-to-tail connected flight is nearly constant but declines slightly in frequency due to the trailing aircraft that fly in the wake of the leading aircraft, causing the roll damping to decline. In addition, the pitch damping of tip-to-tail configurations increase in a similar manner to the roll damping in wing-tip-to-wing-tip connected flight. This increase in pitch damping seen in tip-to-tail connected flight coupled with the quadratic increase in pitch inertia causes the longitudinal modes to become critically damped and even unstable.

Meta aircraft also introduces complex flexible modes, which increase in complexity as the number of aircraft connected is increased. It is found that these flexible modes are largely dependent on the connection parameters of the joint and the aerodynamics of the aircraft, which can alter these modes. These flexible modes are a consequence of relative rotation about the yaw, pitch, and roll axes. It was shown through simulation that the natural frequency and damping can be tuned specifically by changing the rotational stiffness and damping at the joint along each axis, highlighting the decoupled nature of each flexible mode.

\section{References}

[1] Vassilvitskii, S., Yim, M., and Suh, J., "A Complete, Local and Parallel Reconfiguration Algorithm for Cube Style Modular Robots," Proceedings of the International Conference on Robotics and Automation, Vol. 1, Washington, D.C., May 2002, pp. 117-122.
[2] White, P., Zykov, V., Bongard, J., and Lipson, H., "Three Dimensional Stochastic Reconfiguration of Modular Robots," Proceedings of the 2006 Robotics: Science and Systems Conference, MIT Press, Cambridge, MA, 2005, pp. 161-168.

[3] Yim, M., Zhang, Y., and Duff, D., "Modular Robots," IEEE Spectrum, Vol. 39, No. 2, Feb. 2002, pp. 30-34.

[4] Casal, A., and Yim, M., "Self-Reconfiguration Planning for a Class of Modular Robots," Proceedings of SPIE International Symposium on Intelligent Systems and Advanced Manufacturing, Vol. 3839, Sept. 1999, pp. 246-257.

[5] Anderson, C. E. B., "Dangerous Experiments: Wingtip Coupling at 15,000 Feet," Flight Journal, Vol. 5, No. 6, Dec. 2000, p. 64.

[6] Magill, S., "Compound Aircraft Transport: Wing Tip-Docking Compared to Formation Flight," Ph.D. Dissertation, Dept. of Mechanical and Aerospace Engineering, Virginia Polytechnic Inst. and State Univ., Blacksburg, VA, 2002.

[7] Moore, J., and Maddalon, D., "Multi-Body Transport Concept," AIAA 2nd International Very Large Vehicles Conference, AIAA Paper 19820810, May 1982.

[8] Gibbs, J., Vlachos, P., and Telionis, D., "Experimental Determination of Lift and Lift Distributions for Wings in Formation Flight," 43rd AIAA Aerospace Sciences Meeting and Exhibit, AIAA Paper 2005-0843, Jan. 2005.

[9] Magill, S., Schetz, J., and Mason, W., "Compound Aircraft Transport: A Comparison of Wing Tip-Docked and Close-Formation Flight," 41st Aerospace Sciences Meeting and Exhibit, AIAA Paper 2003-0607, Jan. 2003.

[10] Oung, R., Bourgault, F., Donovan, M., and D'Andrea, R., "The Distributed Flight Array," Proceedings of the IEEE International Conference on Robotics and Automation, IEEE Publ., Piscataway, NJ, May 2010, pp. 601-607.

[11] Fowler, J., and D'Andrea, R., "Distributed Control of Close Formation Flight," Proceedings of the IEEE Conference on Decision and Control, Vol. 3, IEEE Publ., Piscataway, NJ, Dec. 2002, pp. 2972-2977.

[12] "Aurora's Odysseus," Aurora Flight Sciences, Manassas, VA, 2014, http://www.aurora.aero/Development/Odysseus.aspx [retrieved $13 \mathrm{Feb}$. 2014].

[13] Etkin, B., Dynamics of Atmospheric Flight, Dover, New York, 2000, pp. 121-152.

[14] Jung, D., "Hierarchical Path Planning and Control of a Small FixedWing UAV: Theory and Experimental Validation," Ph.D. Dissertation, Dept. of Aerospace Engineering, Georgia Inst. of Technology, Atlanta, GA, 2007.

[15] Anderson, D., Fundamentals of Aerodynamics, 4th ed., McGraw-Hill Series in Aeronautical and Aerospace Engineering, McGraw-Hill, New York, 2007, pp. 307-316, 342-348.

[16] Bertin, J., and Smith, M., Aerodynamics for Engineers, 3rd ed., Prentice-Hall, Upper Saddle River, NJ, 1998, pp. 291-311.

[17] Selig, M., "Modeling Full-Envelope Aerodynamics of Small UAVs in Realtime," AIAA Atmospheric Flight Mechanics Conference, AIAA Paper 2010-7635, Aug. 2010.

[18] Anderson, J., Corda, S., and Van Wie, D., "Numerical Lifting Line Theory Applied to Drooped Leading-Edge Wings Below and Above Stall," Journal of Aircraft, Vol. 17, No. 12, 1980, pp. 898-904.

[19] Phillips, W., Mechanics of Flight, 2nd ed., Wiley, Hoboken, NJ, 2010, pp. 715-952. 